\title{
A microbial population dynamics model for the Acetone-Butanol-Ethanol fermentation process
}

\author{
Helena González-Peñas ${ }^{1}$, Thelmo Lu-Chau ${ }^{1}$, Juan Manuel Lema ${ }^{1}$, and Jorge Rodríguez² \\ ${ }^{1}$ Universidade de Santiago de Compostela \\ ${ }^{2}$ Khalifa University, Masdar Institute Campus
}

May 5, 2020

\begin{abstract}
Transient product spectrum during batch Acetone-Butanol-Ethanol fermentation is known to evolve in response to $\mathrm{pH}$-dependent Clostridium acetobutylicum metabolic variations. Although previous works have evidenced the coexistence of distinct cell subpopulations, this culture heterogeneity has been neglected in ABE modeling to date. Here, a dynamic model for ABE fermentation based on bacterial population dynamics was developed under the hypothesis of the existence of a specific solventogenic phenotype. By proposing a simplified metabolic network, the metabolic switches are described, triggered by the partial conversion of acid vegetative cells into solventogenic and sporulating subpopulations. The proposed model succeeds in adequately describing the evolution of all extracellular metabolites in a batch culture both at free and controlled pH. Since the model is controlled by cell population dynamics with no time dependent variable it can be directly applied to alternative flow (e.g. continuous) regimes allowing for its application to a broader range of operating conditions.
\end{abstract}

\section{Hosted file}

ABE-Model-HGP-21Feb2020.docx available at https://authorea.com/users/299627/articles/429219a-microbial-population-dynamics-model-for-the-acetone-butanol-ethanol-fermentationprocess 
figures/Figure-1/Figure-1-eps-converted-to.pdf 
figures/Figure-2/Figure-2-eps-converted-to.pdf 
figures/Figure-3/Figure-3-eps-converted-to.pdf 
figures/Figure-4/Figure-4-eps-converted-to.pdf 
figures/Figure-5/Figure-5-eps-converted-to.pdf 
figures/Figure-6/Figure-6-eps-converted-to.pdf 